\title{
Wnt-5a Promotes Neural Development and Differentiation by Regulating CDK5 via $\mathrm{Ca}^{2+} /$ Calpain Pathway
}

\author{
Yang Shu ${ }^{a, b}$ Min Xiang ${ }^{c}$ Pei Zhang ${ }^{a}$ Guangjian Qi ${ }^{a} \quad$ Feng $\mathrm{He}^{\mathrm{a}}$ \\ Qian Zhang $^{a}$ Zheng Zhang ${ }^{a}$ Zexi Lva Xiang Peng ${ }^{a}$ Hongwei Cai ${ }^{a}$ \\ Bo Tian ${ }^{\mathrm{a} d}$
}

aDepartment of Neurobiology, Tongji Medical School, Huazhong University of Science and Technology, Wuhan, 'Department of Central laboratory, Affiliated Hospital of Jiangsu University, Jiangsu University, Zhenjiang, 'Department of Clinical laboratory, Affiliated Hospital of Jiangsu University, Jiangsu University, Zhenjiang, ${ }^{d}$ Institute for Brain Research, Huazhong University of Science and Technology, Wuhan, China

\section{Key Words}

Wnt signaling - Cyclin-dependent kinase 5 - $\mathrm{Ca}^{2+} /$ calpain signaling - Development • Differentiation

\begin{abstract}
Background/Aims: The Wnt signaling pathway has essential functions in the central nervous system, where it regulates the major physiological functions of neurons, including development, differentiation, and plasticity. Wnt signaling controls these cellular events; however, how Wnt pathways integrate into a coherent developmental program remains unclear. Methods: The expression and secretion of different WNT ligands (Wnt-1, Wnt-3a, Wnt-4, Wnt-5a, Wnt-11), and the levels and activities of cyclin-dependent kinases (CDK2, CDK4, CDK6/cyclin D, cyclin E) or CDK5 (CDK5/p35 and p25) were measured in Rat cortex at different embryonic stages, and in RA/BDNF-induced differentiated SH-SY5Y cell model, by Quantitative real-time PCR (qPCR), western blotting, ELISA, and in vitro CDK5 kinase assays. MAP2-BrdU double staining was used to assess cell differentiation and cell cycle exit in an RA/BDNF-induced differentiated $\mathrm{SH}-\mathrm{SY} 5 \mathrm{Y}$ cell model. The effects of $\mathrm{CDK} 5$ and $\mathrm{Ca}^{2+} /$ calpain signaling were assessed using specific chemical inhibitors. Results: We found that Wnt-1 was unchanged and Wnt-3a was attenuated, whereas Wnt-4, Wnt-5a, and Wnt-11 were markedly up-regulated, during the development of neurons and differentiated SH-SY5Y cells. Simultaneously, the activity of CDK5 was elevated. Furthermore, we describe crosstalk between non-canonical Wnt signaling and CDK5 in the development of neurons and differentiated SH-SY5Y cells. Wnt-5a, a noncanonical Wnt ligand, regulated CDK5 via $\mathrm{Ca}^{2+} /$ calpain signaling in both neuronal development and differentiation. Inhibition of Wnt-5a diminished CDK5 kinase activity via the $\mathrm{Ca}^{2+} / \mathrm{calpain}^{2}$

Y. Shu and M. Xiang contributed equally to this work

\begin{tabular}{ll}
\hline Bo Tian M.D. \& Ph.D. & Department of Neurobiology, Tongji Medical School, Huazhong University of Science and Technology \\
& 13 Hangkong Road, Wuhan, Hubei (China) \\
& E-Mail tianbo@mails.tjmu.edu.cn
\end{tabular}
\end{abstract}


pathway, thereby attenuating RA-BDNF induced SH-SY5Y cell differentiation. Conclusion: Wnt-5a signaling is a significant regulator of neuronal development and differentiation and upregulates CDK5 kinase activity via $\mathrm{Ca}^{2+} /$ calpain signaling.

(C) 2018 The Author(s)

Published by S. Karger AG, Basel

\section{Introduction}

The roles of Wnt signaling in physiological and pathological processes have been comprehensively studied, including its contributions to cell development, differentiation, proliferation, and cancer [1-5]. Most Wnt signaling can be classified into canonical and noncanonical pathways [6]. A majority of Wnt ligands regulate canonical signaling via Wnt/ $\beta$ catenin pathways, including those mediated by the ligands Wnt- 1 and Wnt-3a $[7,8]$. Other ligands mediate the non-canonical Wnt signaling-independent $W n t / \beta$-catenin pathway, including Wnt-4, Wnt-5a, and Wnt-11 [9-11]. Most Wnt ligands have critical functions that contribute to neuronal differentiation of embryonal carcinoma and embryonic stem cells [12]. Wht also affects several other signaling pathways, including Notch and PI3K/ AKT, whose activities affect neuronal development and differentiation [13]. Canonical Wnt signaling regulates axon genesis and the differentiation of cerebellar granule neuron(CGN) progenitors. Non-canonical Wnt signaling regulates vertebrate development and neurite outgrowth through the planar cell polarity (PCP) and Wnt/Ca ${ }^{2+}$ pathways $[14,15]$; however, canonical Wnt can activate non-canonical pathways, and vice versa, by regulating the same downstream components, and human neural stem cell differentiation can be mediated via switching from canonical to non-canonical Wnt signaling. The molecular mechanisms underlying the effects of non-canonical Wnt signaling in brain developmentand differentiation are currently unclear.

Cyclin-dependent kinase 5 (CDK5) is a proline-directed serine/threonine kinase, which is essential for several neuronal functions $[16,17]$. CDK5 activation requires a coactivator, such as p35 or p39 [18]. Intracellular calcium influx can activate calpain to promote conversion of p35 to p25 [19] and a CDK5/p25 complex phosphorylates substrates to regulate neuronal death, development, and differentiation [20-22]. CDK5 regulatory subunit-associated protein 1-like1 (CDKAL1) regulates adipocyte differentiation through the activation of the Wnt pathway [23]. A novel CDK5 splicing variant (CDK5-SV) may be a negative regulator of Wnt/ $\beta$-catenin signaling [24]. In addition, CDK5 phosphorylates liprin $\alpha 1$ to mediate the neuronal activity-dependent synapse development [25].

Recently, CDK5 and Wnt signaling have been shown to be correlated in different circumstances. Wht signaling and the regulation of CDK5 are critical for the development and differentiation of the nervous system; however, their correlation is seldom reported in this context. In this study, we determined that interaction between Wnt signaling and CDK5/p25 can mediate neuronal development and differentiation, as well as exploring the underlying mechanisms. We showed that Wnt-5a upregulates p25 expression, which elevated CDK5 activity during neuronal development and differentiation of SH-SY5Y cells. Consequently, Wnt-5a promoted activation of CDK5/p25-mediated neuronal development and differentiation of SH-SY5Y cells via regulation of the $\mathrm{Ca}^{2+} /$ calpain signaling. In addition, we demonstrate that Wnt-5a activates CDK5, establishing a link between Wnt signaling and CDK5 kinase activity.

\section{Materials and Methods}

Chemicals and antibodies

Recombinant human/Rat Wnt-5a was acquired from Promega (Madison, WI, USA). EGTA-AM, BAPTA-AM, and the calcium ionophore A23187 were purchased from Sigma-Aldrich (St. Louis, MO, USA). Antibodies used for immunostaining or immunoblotting included the following: CDK5 (Santa Cruz, CA, USA), MAP2 (Cell Signaling Technology, MA, USA), p35 (Santa Cruz, CA, USA), and cleaved $\alpha$-fodrin (Cell Signaling Technology, MA, USA). Wnt-5a neutralizing antibody and anti $\beta$-actin were obtained from Sigma- 


\section{Cellular Physiology Cell Physiol Biochem 2018;51:2604-2615

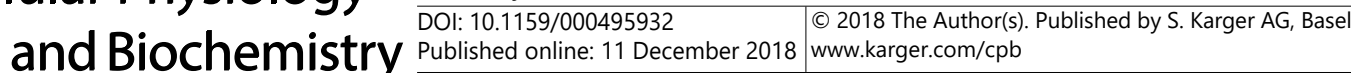

Shu et al.: Wnt-5a Regulates CDK5 Activity in Neurodevelopment

Aldrich (St. Louis, MO, USA). Alexa Fluor 488- and 594-conjugated secondary antibodies were purchased from Invitrogen (Carlsbad, CA, USA).

\section{Cell culture}

Primary cultures of rat cortical neurons were established from an E18 rat. After 3 days, neurons were treated with Wnt-5a or DMSO. SH-SY5Y human neuroblastoma cells were maintained in Dulbecco's modified Eagle's medium (DMEM) supplemented with $10 \%$ heat-inactivated fetal bovine serum (FBS) in $5 \% \mathrm{CO}_{2}$ at $37^{\circ} \mathrm{C}$. DMEM and FBS were obtained from Gibco (Rockville, MD, USA). SH-SY5Y cells were differentiated by incubation with retinoic acid and brain-derived growth factor (RA-BDNF) for 6 days.

\section{Western blotting}

Cells were lysed in RIPA lysis buffer (50 mM Tris-HCl, pH 7.4, 150 mM NaCl, 1\% NP-40, 2 mM EDTA, 10 $\mathrm{mM} \mathrm{NaF}, 2 \mathrm{mM}$ Na3VO4) containing $0.01 \%$ protease inhibitor cocktail. The protein concentrations of lysates were determined using a BCA kit (Beyotime, Nanjing, China). Total lysate aliquots containing an equivalent of $20 \mu \mathrm{g}$ protein per sample were separated by SDS-PAGE and transferred to PVDF membranes. Membranes were blocked using 5\% skim milk or 5\% BSA in TBST. Subsequently, levels of immunodetected protein were analyzed using ECL kits (Amersham, Buckinghamshire, UK).

\section{Enzyme-linked immunosorbent assay (ELISA)}

Cell culture media were transferred to centrifuge tubes and centrifuged to remove debris. Concentrations of various Wnt ligands were detected using their corresponding ELISA kits, according to the manufacturer's instructions (Cusabio, Wuhan, China). The absorbance of samples was measured at $450 \mathrm{~nm}$ using a Multiskan Spectrum (Multiskan, Thermo, USA).

Immunofluorescence, image acquisition, and analyses

Cultured cells were fixed for $1 \mathrm{~h}$ in $4 \%$ paraformaldehyde in phosphate-buffered saline (PBS), then analyzed using a polyclonal rabbit anti-MAP2 primary antibody, and anti-Rat Alexa Fluor 488 and antirabbit Alexa Fluor 594 secondary antibodies. Cells were examined using a Nikon microscope (Nikon, Japan). Subsequently, images were analyzed using Image J Lab software (Bio-Rad, Hercules, CA, USA).

Quantitative real time-polymerase chain reaction ( $q P C R$ )

For quantitative analysis of gene expression involved in Wnt signaling, total RNA was isolated from cortical neuron or SH-SY5Y cells using TRIzol reagent (Invitrogen) and equivalent amounts of cDNA synthesized using the Superscript RT enzyme(Vazyme,china). qPCR was performed using SYBR Green Mix (Takara, Japan), and gene expression level values were normalized to those of the internal control, GAPDH. Primer sequences were as follows:

Wnt-1, forward 5'-GACGATCTTGCCGAAGAG-3' and reverse 5'-CTGTGCGAGAGTGCAA-3' (human), forward 5'-CAAGATCGTCAACCGAGGCT-3' and reverse 5'- GTAGTCGCAGGTGCAGGATT-3' (rat); Wnt-3a, forward 5'- GCCATCGGTGACTTCCTCAA-3' and reverse 5'- TTGAAGTAGGTGTAGCGCGG-3' (human), forward 5'AGGTGCTAACACAGCAGCTTA-3' and reverse 5'- CAGTGAGGAGTACTGGGGTC-3' (rat); Wnt-4, forward 5'- GGCTTCCAGTGGTCAGGATG-3' and reverse 5'- TTGTGGAGGTTCATGAGGGC-3' (human), forward 5'- CTGTTCCACACTGGACTCCC-3' and reverse 5'- ACACCTGCTGAAGAGATGGC-3' (rat); Wnt-5a, forward 5'- AGAAGAAACTGTGCCACTTGTATCAG-3' and reverse 5'- CCTTCGATGTCGGAATTGATACT -3' (human), forward 5'- GAAGGCGAGCTGTCTACCTG-3' and reverse 5'- ACACCTGCTGAAGAGATGGC-3' (rat); Wnt-11, forward 5'- GGATATCCGGCCTGTGAAGG-3' and reverse 5'- GTTGCACTGCCTGTCTTGTG -3' (human), forward 5'- AGACAGGCAGTGCAACAAGA-3' and reverse 5'- GTCCGTGTAGGGGTTGTAGC-3' (rat); GAPDH, forward 5'- ACCCAGAAGACTGTGGATGG-3' and reverse 5'- TTCAGCTCAGGGATGACCTT-3' (human), forward 5'GACATGCCGCCTGGAGAAAC-3' and reverse 5' - AGCCCAGGATGCCCTTTAGT-3' (rat).

\section{CDK5 kinase assay}

Purified Histone $\mathrm{H} 1$ protein $(1.5 \mu \mathrm{g})$ was incubated for $1 \mathrm{~h}$ at $30^{\circ} \mathrm{C}$ with p25/CDK5 in kinase buffer (20 mM MOPS, pH 7.0, $10 \mathrm{mM} \mathrm{MgCl} 2,1 \mathrm{mM}$ DTT, and $20 \mu \mathrm{M}$ sodium orthovanadate) containing $100 \mu \mathrm{M}$ nonradioactive ATP and $5 \mu \mathrm{Ci}[\gamma-32 \mathrm{P}]$ ATP. Reaction mixtures were separated by SDS-PAGE and measured by autoradiography [17]. 


\section{Cellular Physiology Cell Physiol Biochem 2018;51:2604-2615 and Biochemistry \begin{tabular}{l|l} 
DOI: 10.1159/000495932 & $\begin{array}{l}\text { (c) } 2018 \text { The Author(s). Published by S. Karger AG, Basel } \\
\text { www.karger.com/cpb }\end{array}$
\end{tabular} \\ Shu et al.: Wnt-5a Regulates CDK5 Activity in Neurodevelopment}

\section{Statistical analysis}

All data were presented as means \pm standard error of the mean(SEM). Comparisons between two groups were conducted using two-tailed Student's t-tests. Anova was used here for comparing more than two groups. $\mathrm{P}<0.05$ was considered statistically significant. All the experiments were repeated three times excluding certain chance factors.

\section{Results}

During neuronal development, canonical Wht signaling is attenuated, whereas noncanonical Wnt signaling is upregulated

To determine the role of canonical and non-canonical Wnt pathways in cortical development, we first evaluated the expression patterns of Wnt family genes throughout Rat brain development from embryonic day 11.5 to stage P0 (E11.5-P0). Next, we analyzed the expression levels of the canonical (Wnt-1 and Wnt-3a) and non-canonical (Wnt-4, Wnt-5a, and Wnt-11) Wnt ligands by qPCR. Wnt-1 mRNA levels did not alter significantly, while those of Wnt-3a were reduced; however, the mRNA levels of Wnt-4, Wnt-5a, and Wnt-11 increased with progressing embryonic development (Fig. 1a, 1b).

In the RA/BDNF-stimulated SH-SY5Y cell differentiation model, non-canonical Wnt signaling was upregulated at the mRNA and protein levels in differentiated SH-SY5Y cells compared with levels prior to differentiation (Fig. 1c, 1d). Together, these results indicate that non-canonical Wnt signaling is critical for cortical neuronal development and differentiation.

Fig. 1. Expression and secretion of non-canonical Wnt ligands (Wnt-4, Wnt-5 a, and Wnt-11) increase, while those of the canonical Wnt ligands (Wnt-1 and Wnt-3a) decrease during neuronal development and differentiation. (a) qPCR analysis of canonical Wnt signaling ligand expression in the Rat cerebral cortex from embryonic day 11.5 to birth (E11.5 to P0). Wnt-1 and Wnt-3a expression levels are shown through developmental progression. (b) qPCR analysis of non-canonical Wnt signaling ligand expression in the Rat cerebral cortex from E11.5 to P0. Wnt-4, Wnt-5a,

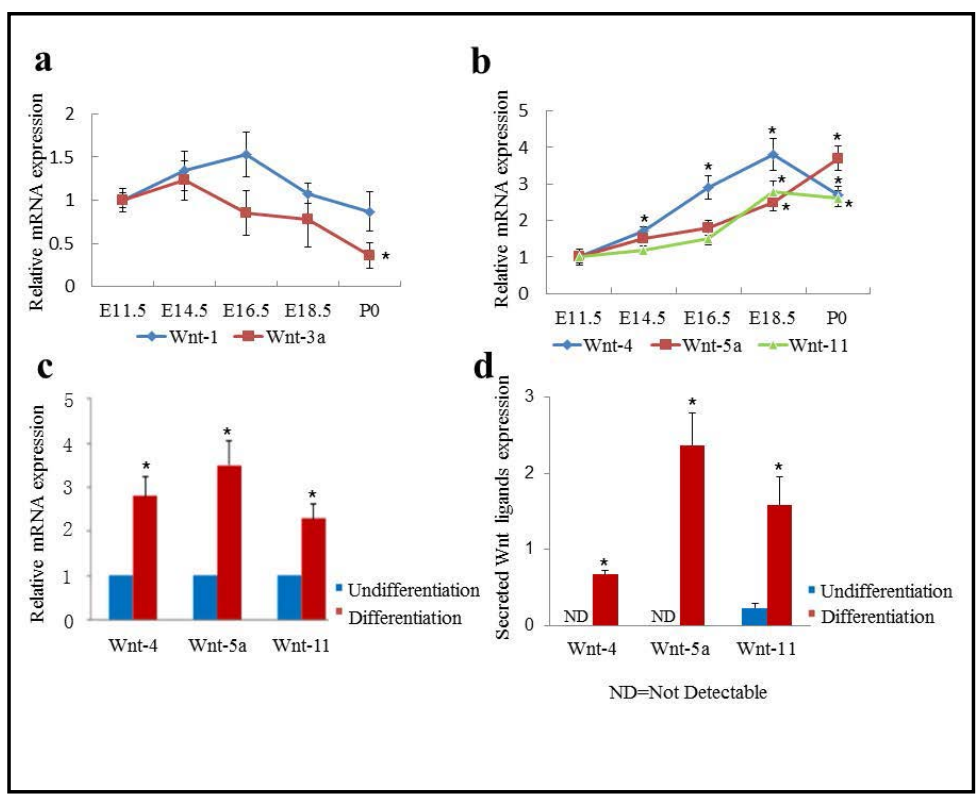
and Wnt-11 expression levels are shown through developmental progression. (c) qPCR analysis of non-canonical Wnt signaling ligands demonstrating in the SH-SY5Y cell model of differentiation. Wnt-4, Wnt-5a, and Wnt-11 expression levels are shown in SH-SY5Y cell differentiated model. (d) ELISA analysis of non-canonical Wnt signaling ligands to compare protein expression between RA/BDNF-stimulated and control SH-SY5Y cell groups. Wnt-4, Wnt-5a, and Wnt-11 expression levels are shown in SH-SY5Y cell differentiated model. *p<0.05; Data were calculated from three repeated experiments; ND, Not Detectable. 
Altered CDKs/cyclins and CDK5/ p25 in neuronal development and differentiation

Next, we conducted experiments to investigate the roles of CDKs/ cyclins. To understand the function of CDKs during neuronal development, we assessed the mRNA levels of CDK2, CDK4, and CDK6 by qPCR analysis and found no significant differences in their levels during cortical neuronal development (Fig. 2a). Therefore, we investigated the cyclin partners of CDK2, CDK4, and CDK6, cyclin D and cyclin E, in the same context and found that expression levels of cyclin D was downregulated and cyclin $\mathrm{E}$ was unaltered (Fig. 2b), indicating that CDKs/cyclins do not have significant roles in cortical cell development.

CDK5 is a unique member of the CDK family, with diverse functions related to nervous system regulation. Therefore, we investigated whether CDK5 can regulate cortical neuronal development and found high levels of p25 expression during neuronal development (Fig. 2c). Moreover, western blotting revealed that CDK5 kinase activity increased with progressing embryonic development and differentiation (Fig. 2d). These data suggested a potential role for CDK5 in cortical neuronal development.

To further evaluate the role of CDK5/p25 in neuronal development, we used the RA/ BDNF-induced SH-SY5Y cell differentiation model. The mRNA levels of CDK2, CDK4, CDK6, and Cyclin E did not differ significantly in these cells before and after differentiation; however, levels of Cyclin D declined dramatically in differentiated SH-SY5Y cells (Fig. 3a, 3b). CDK5 mRNA and protein levels did not differ significantly between the two groups; however, those of p25 increased markedly. In addition, p25, a degradation product of p35, was detected in differentiated SH-SY5Y cells (Fig. 3c, 3d), and CDK5 kinase activity was upregulated (Fig. 3d).

Wnt-5a, but not Wnt-3, Wnt-4, or Wnt-11, directly activates CDK5 kinase activity in neuronal development and differentiation

Our previous results showed that non-canonical Wnt signaling was upregulated during neuronal development and differentiation. Next, we focused on studying the mechanism underlying non-canonical Wnt signaling in this context. We stimulated cortical neurons with different concentrations of various recombinant non-canonical pathway Wnt ligands. The results indicated that CDK5 kinase activity was upregulated in response to stimulation with Wnt-5a; however, no significant changes in CDK5 kinase activity were observed in response to Wnt-3a, Wnt-4, or Wnt-11 (Fig. 4a). Similarly, stimulation with Wnt-5a at various time points demonstrated that the CDK5 kinase activity was upregulated in cortical neurons (Fig. 
Fig. 3. Expression of p35 increased significantly, while there was no change in the levels of CDKs (CDK2, CDK4, CDK5, and CDK6) in the RA/BDNFstimulated SH-SY5Y cell differentiation model. qPCR analysis of cycling CDKs/ cyclins expression in SH-SY5Y cells. (a) and (b). SH-SY5Y cells were stimulated by RA/BDNF and levels of mRNA measured by qPCR. (c) Using the same cell model as (a) and (b), the expression

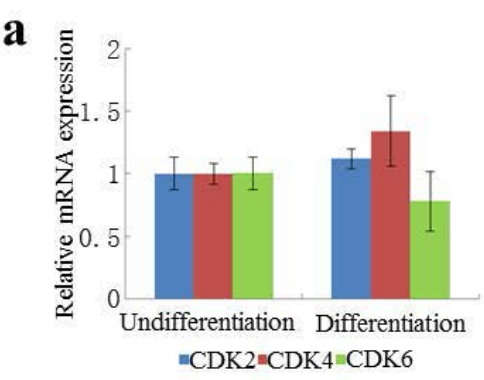

c

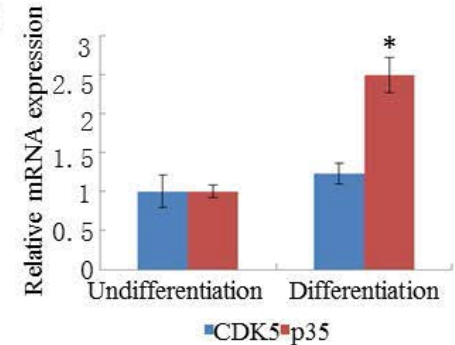

b

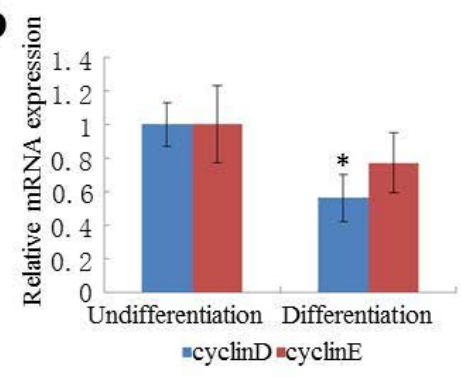

d

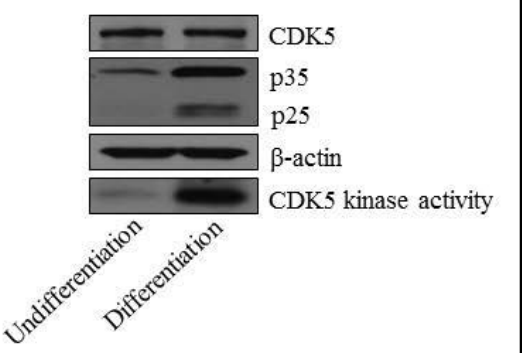
mRNA levels of CDK5 and p35 were measured by qPCR. SH-SY5Y cells stimulated with PBS were used as negative controls. GAPDH was used as a reference gene. (d) Protein expression levels of CDK5 and p35/p25 in SH-SY5Y cells were estimated using western blotting. $\beta$-actin was used as a control. Induction of CDK5 kinase activity by RA/ BDNF in SH-SY5Y cells was evaluated using an in vitro CDK5 phosphorylation assay. * $<<0.05$; Data were calculated from three repeated experiments.

Fig. 4. Non-canonical Wnt-5a upregulated p35 expression and activated CDK5 kinase. E18 embryonic rat cortical neurons were cultured for 3 days in B27 B27/neurobasal medium. Neurons were treated with different concentrations of Wnt3, Wnt-4, Wnt-5a, and Wnt-11, and CDK5 activity was detected by autoradiography, via evaluation of Histone $\mathrm{H} 1$ phosphorylation, which was used as a CDK5

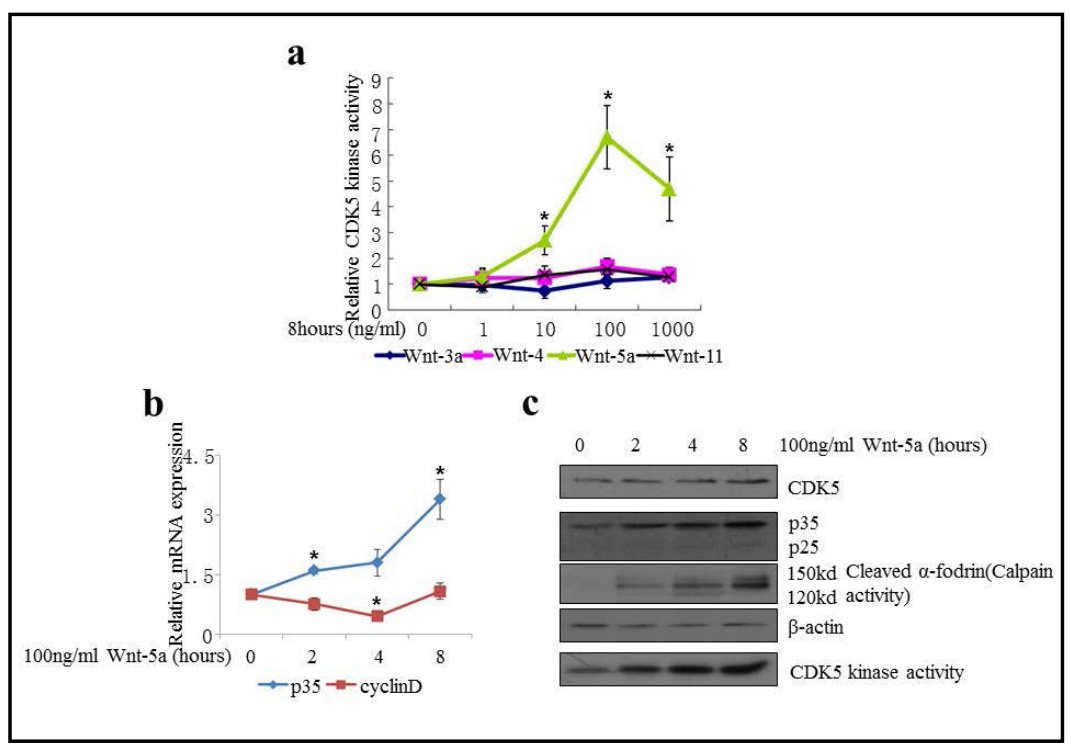
substrate for the CDK5

kinase reaction. Data are representative of a single independent experiment. (a) Wnt-5a (10, 100 or 1000 $\mathrm{ng} / \mathrm{mL}$ ) can activate CDK5. (b) qPCR results showing p35 and cyclin D levels in Wnt-5a-treated neurons. GADPH transcript levels are presented as controls. (c) Wnt-5a increases the levels of p25 and cleaved $\alpha$-fodrin expression from 0-8 h after Wnt-5a treatment in neurons. p25 expression (panel 2); Cleaved $\alpha$-fodrin expression (panel 3); CDK5 activity (panel 5). ${ }^{*} \mathrm{p}<0.05$; Values were means \pm SEM of three individual experiments.

\section{KARGER}


4c). mRNA and protein levels of p35 were also upregulated in response to Wnt-5a (Fig. 4b, $4 c)$. In addition, cleaved $\alpha$-fodrin, a substrate of calpain, appeared on Wnt-5a stimulation (Fig. 4c); therefore, we deduced that calpain participates in Wnt-5a/CDK5 signaling in this context.

\section{Wnt-5a activates $C D K 5$ through the $\mathrm{Ca}^{2+} /$ calpain pathway}

In the present study, enhanced CDK5 kinase activity in neurons was mediated by Wnt$5 \mathrm{a}$; however, the mechanisms triggering CDK5 kinase activity remain unknown. Therefore, we sought to elucidate how Wnt-5a induces CDK5 kinase activity. Recent reports have highlighted that $\mathrm{Ca}^{2+} /$ calpain signaling activity is a significant downstream target of Wnt-5a stimulation. Moreover, CDK5 signaling is regulated by $\mathrm{Ca}^{2+} /$ calpain activity, which degrades p35 into p25, a highly active molecular chaperone involved in CDK5 activation; therefore, we hypothesized that Wnt-5a activates CDK5 through the $\mathrm{Ca}^{2+} /$ calpain pathway. CDK5 kinase activity was upregulated by exposure of cultured cortical neurons to a calcium ionophore at different time points $(0-8 \mathrm{~h}$ ) (Fig. $5 \mathrm{a})$. In addition, Wht-5a-mediated upregulation of CDK5

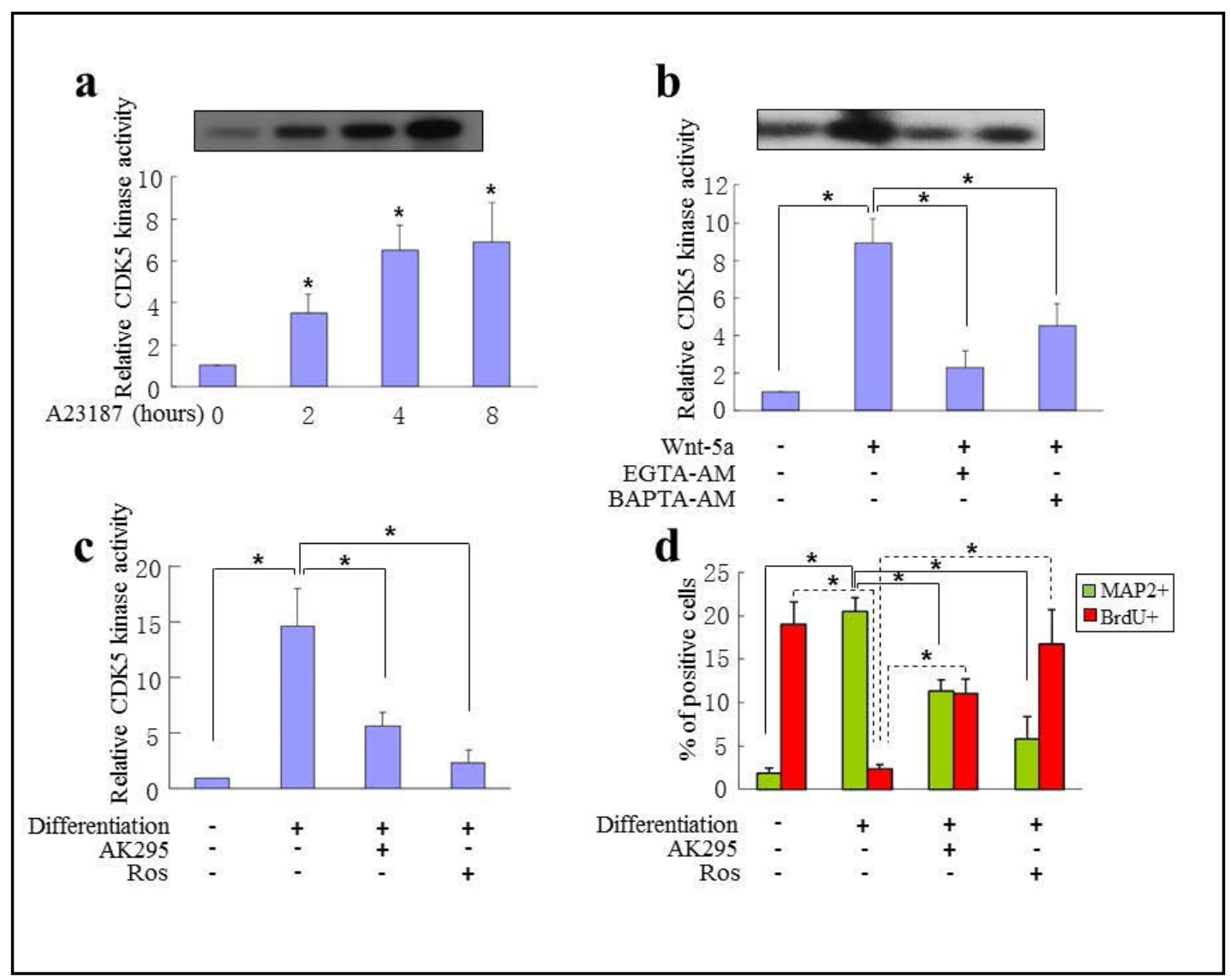

Fig. 5. Wnt-5a activates CDK5 through the Ca2+/calpain pathway. (a) The effect of calcium ionophore on the activity of CDK5. Primary neurons were stimulated by A23187 (calcium ionophore) for different periods of time (0, 2, 4, and $8 \mathrm{~h}$ ). (b) Schematic representation of CDK5 kinase activity in response to Wnt-5a, EGTAAM, or BATPA-AM. Primary neurons were stimulated by Wnt-5a, EGTA-AM, or BATPA-AM. (c) Schematic representation of CDK5 kinase activity in response to treatment with AK295 and Ros during SH-SY5Y cell differentiation. (d) Immunofluorescence analysis using the markers MAP2+ and BrdU+ following AK295 or Ros treatment during SH-SY5Y cell differentiation (mature differentiated neurons are marked by MAP2+, while neurons in $\mathrm{S}$ phase or undergoing division are marked by BrdU+). ${ }^{*} \mathrm{p}<0.05$; Data were representative of multiple experiments ( $n=3$ ); A23187, Calcium Ionophore; EGTA-AM and BATPA-AM, Calcium Chelators; AK295, Calpain Inhibitor; Ros, CDK5 Inhibitor.

\section{KARGER}


kinase activity was reduced by treatment with different calcium chelators (EGTA-AM and BAPTA-AM) (Fig. 5b). Consistent with these results, we found that AK295, a calpain inhibitor, and Roscovitine (Ros) attenuated CDK5 kinase activity in differentiated SH-SY5Y cells (Fig. 5c). Moreover, numbers of differentiated SH-SY5Y cells were markedly reduced by AK295 or Ros in the RA/BDNF model (Fig. 5d).

\section{Blocking Wnt-5a attenuates RA-BDNF-induced SH-SY5Y cell differentiation}

Our previous results demonstrated that Wnt-5a could activate CDK5 kinase activity. Next, we examined whether Wnt-5a regulated neuronal differentiation through its activation of CDK5. We firstly tested that Wnt-5a downregulation using a Wnt neutralizing antibody could inhibit CDK5 kinase activity (Fig. 6a). The antibody could partially block Wnt-5a binding to Wnt and suppress SH-SY5Y cell differentiation, which was also inhibited by Box5 (a Wnt-5a antagonist), demonstrating a critical role for Wnt-5a in BDNF-induced SH-SY5Y cell differentiation (Fig. 6b, 6c). We also constructed an siRNA that specifically silenced Wnt-5a expression in SH-SY5Y cells and confirmed its knockdown efficiency in vivo (Fig. $6 \mathrm{~d})$. Our results verified that activation of CDK5 could be inhibited and the proportion of differentiated SH-SY5Y cells reduced by treatment with Wnt-5a-specific siRNA (Fig. 6e, 6f). Hence, Wnt-5a appears to be a critical factor in SH-SY5Y cell maturation. Collectively, these results indicate that Wnt-5a supports SH-SY5Y cell differentiation.

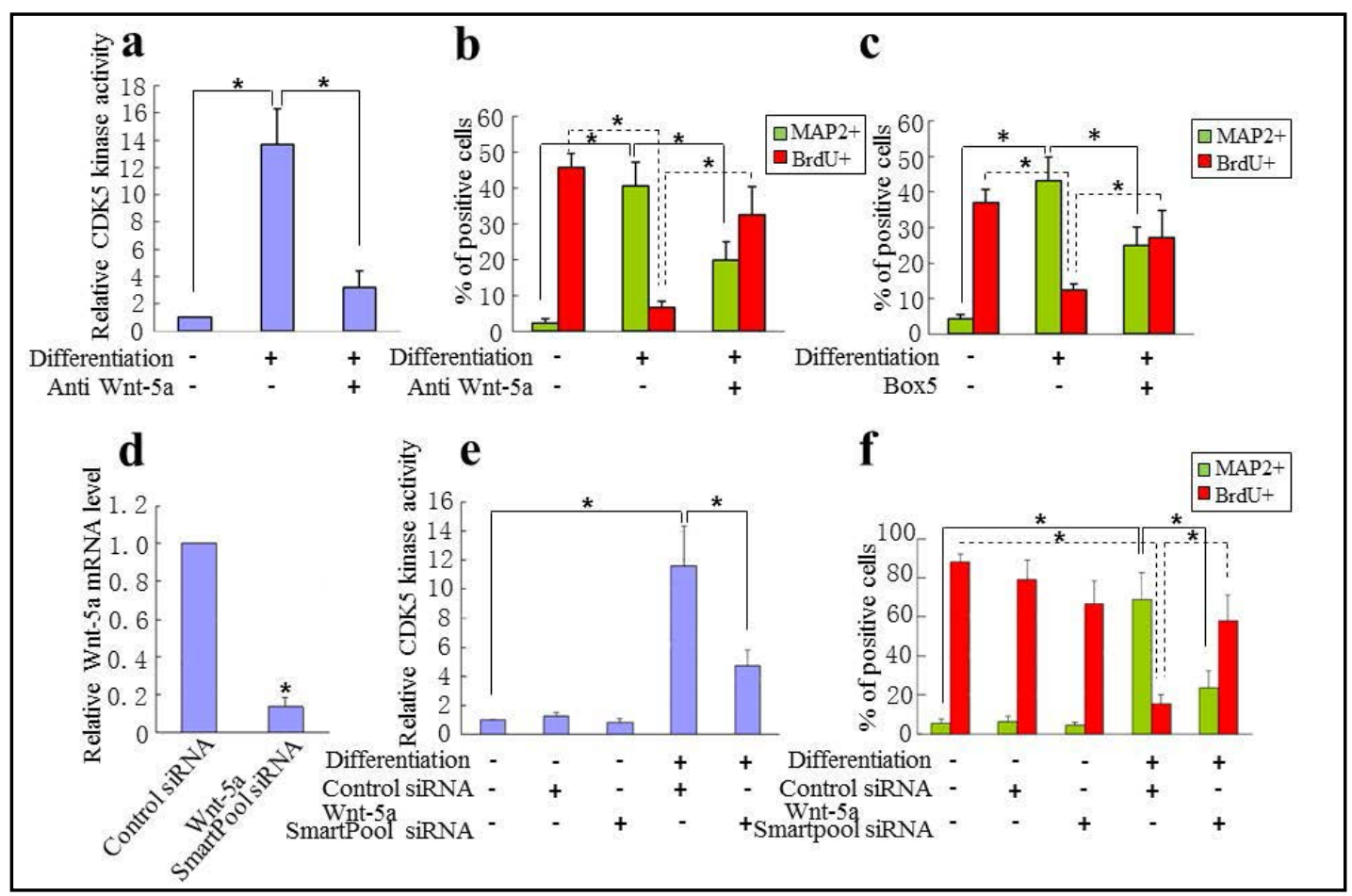

Fig. 6. Blocking of Wnt-5a attenuates RA-BDNF-induced SH-SY5Y cells differentiation. (a) CDK5 kinase activity assay using SH-SY5Y cells cultured in the presence or absence of Wnt-5a neutralizing antibody after RA and BDNF stimulation. (b) and (c) Immunofluorescence-positive cells stained with MAP2+ and BrdU+ antibody in SH-SY5Y cells cultured in the presence or absence of Wnt-5a neutralizing antibody and Wnt-5a antagonist (BOX5) after RA and BDNF stimulation, respectively. (d) Control or Wnt-5a smartPool siRNAs were transfected into SH-SY5Y cells, and the levels of Wnt-5a mRNA analyzed by qPCR. (e) Treatment with the Wnt-5a smartPool siRNA led to a decrease in CDK5 kinase activity in SH-SY5Y cells after RA and BDNF stimulation. (f) Immunofluorescence analysis of positive cells stained with MAP2+ and BrdU+ antibodies after treatment with control siRNA or Wnt-5a smartPool siRNA in SH-SY5Y cells after RA and BDNF stimulation. ${ }^{*} \mathrm{p}<0.05$, mean $\pm \mathrm{SEM}$ of three independent experiments. 


\section{Cellular Physiology Cell Physiol Biochem 2018;51:2604-2615 \\ and Biochemistry Published \begin{tabular}{l|l} 
DOI: 10.1159/000495932 & $\begin{array}{l}\text { @ } 2018 \text { The Author(s). Published by S. Karger AG, Basel } \\
\text { www.karger.com/cpb }\end{array}$
\end{tabular}}

Shu et al.: Wnt-5a Regulates CDK5 Activity in Neurodevelopment

\section{Discussion}

Previous studies have demonstrated a significant role for Wht signaling in neuronal development and differentiation; however, little is known about $\beta$-catenin-independent Wnt signaling in this context $[26,27]$. Among the effects of Wnt proteins on the nervous system, the $W n t / \beta$-catenin pathway has a crucial role in cell proliferation and differentiation, while non-Wnt/ $\beta$-catenin signaling is key for regulation of cell polarity and motility [28]. Wnt/ $\beta$-catenin signaling also controls postnatal Rat epididymal development, while it has no influence on epithelial cell differentiation [29]. Low-affinity nerve growth factor receptor (LNGFR) targets the Wnt/ $\beta$-catenin pathway and promotes osteogenic differentiation in rat ecto-mesenchymal stem cells [30]. Celecoxib-induced inhibition of neurogenesis in the fetal frontal cortex is attenuated by curcumin via the Wnt/ $\beta$-catenin pathway; however, the expression patterns and functions of Wnt proteins in neuronal development and differentiation have yet to be elucidated. Recently, the non-Wnt/ $\beta$-catenin pathway was identified as involved in embryonic development $[31,32]$. The data presented in this report demonstrate the role of Wnt-5a signaling in the regulation of neuronal development and differentiation for the first time.

Individual Wnt proteins can initiate multiple downstream signaling events [33]. Several Wnt ligands, including Wnt-4a, Wnt-5a, Wnt-7a, Wnt-11, and Wnt-16, can activate the noncanonical Wnt/ $\beta$-catenin pathway. Wnt-5a has been extensively studied [34]; it is required for notochord cell intercalation through the Wnt/PCP pathway in the ascidian Halocynthia roretzi [35] and can also activate the Wnt/ $\beta$-catenin pathway under certain conditions $[36,37]$. The Wnt/PCP pathway activates JUK/Rho, while the Wnt/Ca ${ }^{2+}$ pathway increases intracellular calcium concentrations and activates protein kinase $\mathrm{C}$ (PKC). Wnt-5a can both activate and repress Wnt/ $\beta$-catenin signaling during Rat embryonic development [37]. It also regulates the expression of MESP1 and cardiomyocyte differentiation via non-Wnt/ $\beta$-catenin signaling [38]. Wnt-5a is expressed at an early of in vitro osteogenesis differentiation [39]. Together, these experiments show that Wnt-5a can activate multiple receptors to influence various cellular processes during embryonic development. Therefore, we hypothesized that Wnt-5a may be important for neuronal development and differentiation. Finally, we demonstrated a relationship between Wnt-5a and CDK5 activation, where Wnt-5a regulated CDK5 activation through modulation of the $\mathrm{Ca}^{2+} /$ calpain pathway.

Previous studies have demonstrated that regulation of CDK5 is essential for early neuronal development and differentiation. CDK5 activation, by conversion of p35 to p25, is triggered by calpain activation, which is mediated by intracellular $\mathrm{Ca}^{2+}$ levels [40]. Here, we studied the effect of CDK5-regulated Wnt signaling. In the Wnt/PKC-Ca ${ }^{2+}$ pathway, PKC triggers $\mathrm{Ca}^{2+}$ release and activates the transcription factor NFAT (nuclear factor of activated T-cells) [41]. Wnt/PKC-Ca ${ }^{2+}$ is activated via Wnt-5a; therefore, we hypothesize that Wnt5 a increases intracellular $\mathrm{Ca}^{2+}$ levels to activate calpain in SH-SY5Y cells stimulated by RABDNF. Furthermore, we provide evidence for the involvement of Wnt-5a-activated CDK5 in the regulation of neuronal development and differentiation.

Calpain, a $\mathrm{Ca}^{2+}$-mediated protease, is regulated by a rarely reported Wnt signaling pathway. In SW480 cells, calpain is negatively regulated by $\beta$-catenin signaling, which is downregulated by Wnt-5a. Thus, it would be of interest to verify the correlation between Wnt-5a and $\beta$-catenin. Here, we demonstrate that the upregulation of Wnt-5a is a key factor in activation of CDK5 kinase through regulation of $\mathrm{Ca}^{2+}$-mediated calpain activity. These results suggest that CDK5 is a key player downstream of Wnt-5a. Blocking calpain activity reduces Wnt-5a-mediated CDK5 kinase activity; therefore, our investigation of Wnt-5a in neuronal development and differentiation reveal critical mechanisms and enrich our understanding of CDK5/p25 functions. Several recent studies have shown that Wnt-5a and ROR2 (an orphan tyrosine kinase receptor) can regulate $\mathrm{Ca}^{2+} /$ calpain activity in melanoma cells. Our future investigations will focus on the Wnt-5a/ROR2 pathway. 
In the current study, we present data revealing the effects of Wnt-5a on the central nervous system, demonstrating that Wnt-5a promotes neuronal development and SH-SY5Y cell differentiation. Importantly, we also identified the cellular mechanism underlying Wnt$5 \mathrm{a} / \mathrm{Ca}^{2+}$-calpain/CDK5-mediated regulation of neuronal development and differentiation.

\section{Acknowledgements}

This work was supported financially by the National Natural Science Foundation of China (No. 81601043 to Y. S. and No. 31371384 and 31571044 to B. T.), the Natural Science Foundation of Jiangsu Province (No. BK 20160548 to Y. S.), the Major Social Development Project of Zhenjiang city (No. SH2016032 to Y. S.), and Doctor startup Fund Program (No. JDFYRC-2015005 to Y. S.).

\section{Disclosure Statement}

All the authors declare no competing interests.

\section{References}

1 Wang L, Dehm SM, Hillman DW, Sicotte H, Tan W, Gormley M, Bhargava V, Jimenez R, Xie F, Yin P, Qin S, Quevedo F, Costello BA, Pitot HC, Ho T, Bryce AH, Ye Z, Li Y, Eiken P, Vedell PT et al.: A Prospective GenomeWide Study of Prostate Cancer Metastases Reveals Association of Wnt Pathway Activation and Increased Cell Cycle Proliferation with Primary Resistance to Abiraterone Acetate-Prednisone. Ann Oncol DOI: 10.1093/annonc/mdx689.

-2 Wang W, Pan Q Fuhler GM, Smits R, Peppelenbosch MP: Action and function of Wnt/ $\beta$-catenin signaling in the progression from chronic hepatitis C to hepatocellular carcinoma. J Gastroenterolo 2017;52:419-431.

-3 Fasihi A, Soltani BM, Atashi A, Nasiri S: Introduction of hsa-miR-103a and hsa-miR-1827 and hsa-miR-137 as new regulators of Wnt signaling pathway and their relation to colorectal carcinoma. J Cell Biochem 2018;119:5104-5117.

4 Wang D, Liu C, Wang Y, Wang W, Wang K, Wu X, Li Z, Zhao C, Li L, Peng L: Impact of miR-26b on cardiomyocyte differentiation in P19 cells through regulating canonical/non-canonical Wnt signalling. Cell Prolif;DOI: $10.1111 /$ cpr.12371.

5 Qu R, Hao S, Jin X, Shi G, Yu Q, Tong X, Guo D: MicroRNA-374b reduces the proliferation and invasion of colon cancer cells by regulation of LRH-1/Wnt signaling. Gene 2017;642:354-361.

6 Bocchi R, Egervari K, Carol-Perdiguer L, Viale B, Quairiaux C, De Roo M, Boitard M, Oskouie S, Salmon P, Kiss JZ: Perturbed Wnt signaling leads to neuronal migration delay, altered interhemispheric connections and impaired social behavior. Nat Commun 2017;8:1158.

7 Mussmann C, Hubner R, Trilck M, Rolfs A, Frech MJ: HES5 is a key mediator of Wnt -3a-induced neuronal differentiation. Stem cells 2014;23:1328-1339.

-8 Zhang J, Kang N, Yu X, Ma Y, Pang X: Radial Extracorporeal Shock Wave Therapy Enhances the Proliferation and Differentiation of Neural Stem Cells by Notch, PI3K/AKT, and beta-catenin Signaling. Sci Rep 2017;7:15321.

-9 Subashini C, Dhanesh SB, Chen CM, Riya PA, Meera V, Divya TS, Kuruvilla R, Buttler K, James J: Wnt5a is a crucial regulator of neurogenesis during cerebellum development. Sci Rep 2017;7:42523.

10 Jang S, Cho HH, Park JS, Jeong HS: Non-canonical Wnt mediated neurogenic differentiation of human bone marrow-derived mesenchymal stem cells. Neurosci Lett 2017;660:68-73.

11 Wang L, Qing L, Liu H, Liu N, Qiao J, Cui C, He T, Zhao R, Liu F, Yan F, Wang C, Liang K, Guo X, Shen YH, Hou $\mathrm{X}$, Chen L: Mesenchymal stromal cells ameliorate oxidative stress-induced islet endothelium apoptosis and functional impairment via Wnt4- $\beta$-catenin signaling. Stem Cell Res Ther 2017;8:188.

12 Kikuchi A, Yamamoto H, Sato A, Matsumoto S: New insights into the mechanism of Wnt signaling pathway activation. Int Rev Cell Mol Biol 2011;291:21-71. 


\section{Cellular Physiology Cell Physiol Biochem 2018;51:2604-2615

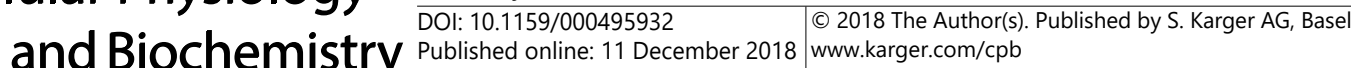

Shu et al.: Wnt-5a Regulates CDK5 Activity in Neurodevelopment

13 Uribe-Etxebarria V, Luzuriaga J, Garcia-Gallastegui P, Agliano A, Unda F, Ibarretxe G: Notch/ Wnt crosssignalling regulates stemness of dental pulp stem cells through expression of neural crest and core pluripotency factors. Eur cells Mater 2017;34:249-270.

14 Veeman MT, Axelrod JD, Moon RT: A second canon. Functions and mechanisms of beta-catenin-independent Wnt signaling. Dev Cell 2003;5:367-377.

15 Selvaraj P, Huang JS, Chen A, Skalka N, Rosin-Arbesfeld R, Loh YP: Neurotrophic factor-alpha1 modulates NGF-induced neurite outgrowth through interaction with Wnt-3a and Wnt-5a in PC12 cells and cortical neurons. Mol Cell Neurosci 2015;68:222-233.

16 Lai KO, Ip NY: Cdk5: a key player at neuronal synapse with diverse functions. Mini Rev Med Chem 2015;15:390-395.

17 Shu Y, Ming J, Zhang P, Wang Q, Jiao F, Tian B: Parkinson-Related LRRK2 Mutation R1628P Enables Cdk5 Phosphorylation of LRRK2 and Upregulates Its Kinase Activity. PloS One 2016;11:e0149739.

18 Malumbres M: Cyclin-dependent kinases. Genome Biol 2014;15:122.

19 Giese KP: Generation of the Cdk5 activator p25 is a memory mechanism that is affected in early Alzheimer's disease. Front Mol Neurosci 2014;7:36.

20 Wilkaniec A, Czapski GA, Adamczyk A: Cdk5 at crossroads of protein oligomerization in neurodegenerative diseases: facts and hypotheses. J Neurochem 2016;136:222-233.

21 Shah K, Lahiri DK: A Tale of the Good and Bad: Remodeling of the Microtubule Network in the Brain by Cdk5. Mol Neurobiol 2017;54:2255-2268.

22 Kawauchi T: Cdk5 regulates multiple cellular events in neural development, function and disease. Dev Growth Differ 2014;56:335-348.

23 Take K, Waki H, Sun W, Wada T, Yu J, Nakamura M, Aoyama T, Yamauchi T, Kadowaki T: CDK5 Regulatory Subunit-Associated Protein 1-like 1 Negatively Regulates Adipocyte Differentiation through Activation of Signaling Pathway. Sci Rep 2017;7:7326.

-24 Li Q, Liu X, Zhang M, Ye G, Qiao Q Ling Y, Wu Y, Zhang Y, Yu L: Characterization of a novel human CDK5 splicing variant that inhibits /beta-catenin signaling. Mol Biol Rep 2010;37:2415-2421.

25 Huang H, Lin X, Liang Z, Zhao T, Du S, Loy MMT, Lai KO, Fu AKY, Ip NY: Cdk5-dependent phosphorylation of liprinalpha1 mediates neuronal activity-dependent synapse development. Proc Natl Acad Sci U S A 2017;114:E6992-E7001.

-26 Suebsoonthron J, Jaroonwitchawan T, Yamabhai M, Noisa P: Inhibition of signaling reduces differentiation and induces sensitivity to doxorubicin in human malignant neuroblastoma SH-SY5Y cells. Anticancer drugs 2017;28:469-479.

27 Lange C, Mix E, Rateitschak K, Rolfs A: Wnt signal pathways and neural stem cell differentiation. Neurodegener Dis 2006;3:76-86.

28 Xiao Q Chen Z, Jin X, Mao R, Chen Z: The many postures of noncanonical Wnt signaling in development and diseases. Biomed Pharmacother 2017;93:359-369.

29 Kumar M, Tanwar PS: Canonical Wnt/ $\beta$-catenin signaling regulates postnatal Rat epididymal development but does not affect epithelial cell differentiation. Endocrinology 2017;158:4286-4299.

-30 Li G, Liu J, Wang Y, Yang K, Zhao M, Xiao Y, Wen X, Liu L: LNGFR targets the Wnt/ $\beta$-catenin pathway and promotes the osteogenic differentiation in rat ectomesenchymal stem cells. Sci Rep 2017;7:11021.

-31 Narendra Talabattula VA, Morgan P, Frech MJ, Uhrmacher AM, Herchenroder O, Putzer BM, Rolfs A, Luo J: Non-canonical pathway induced by Wnt3a regulates beta-catenin via Pyk2 in differentiating human neural progenitor cells. Biochem Biophys Res Commun 2017;49:40-46.

-32 Clark CE, Nourse CC, Cooper HM: The tangled web of non-canonical Wnt signalling in neural migration. NeuroSignals 2012;20:202-220.

-33 Cha SW, Tadjuidje E, Tao Q, Wylie C, Heasman J: Wnt5a and Wnt11 interact in a maternal Dkk1-regulated fashion to activate both canonical and non-canonical signaling in Xenopus axis formation. Development 2008;135:3719-3729.

34 Kikuchi A, Yamamoto H, Sato A, Matsumoto S: Wnt5a: its signalling, functions and implication in diseases. Acta physiol (Oxf) 2012;204:17-33.

-35 Niwano T, Takatori N, Kumano G, Nishida H: Wnt5 is required for notochord cell intercalation in the ascidian Halocynthia roretzi. Biol Cell 2009;101:645-659. 


\section{Cellular Physiology Cell Physiol Biochem 2018;51:2604-2615

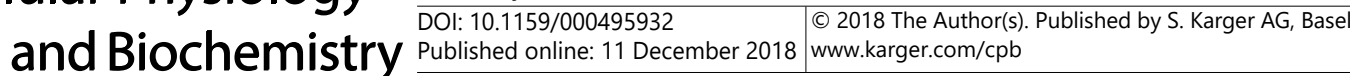

Shu et al.: Wnt-5a Regulates CDK5 Activity in Neurodevelopment

36 Okamoto M, Udagawa N, Uehara S, Maeda K, Yamashita T, Nakamichi Y, Kato H, Saito N, Minami Y, Takahashi N, Kobayashi Y: Noncanonical Wnt5a enhances /beta-catenin signaling during osteoblastogenesis. Sci Rep 2014;4:4493.

-37 van Amerongen R, Fuerer C, Mizutani M, Nusse R: Wnt5a can both activate and repress /beta-catenin signaling during Rat embryonic development. Dev Biol 2012;369:101-114.

-38 Mazzotta S, Neves C, Bonner RJ, Bernardo AS, Docherty K, Hoppler S: Distinctive Roles of Canonical and Noncanonical Wnt Signaling in Human Embryonic Cardiomyocyte Development. Stem cell reports 2016;7:764-776.

-39 Keller KC, Ding H, Tieu R, Sparks NR, Ehnes DD, Zur Nieden NI: Wnt5a Supports Osteogenic Lineage Decisions in Embryonic Stem Cells. Stem cells Dev 2016;25:1020-1032.

40 Park J, Kim B, Chae U, Lee DG, Kam MK, Lee SR, Lee S, Lee HS, Park JW, Lee DS: Peroxiredoxin 5 Decreases Beta-Amyloid-Mediated Cyclin-Dependent Kinase 5 Activation Through Regulation of $\mathrm{Ca}^{2+}$-Mediated Calpain Activation. Antioxid Redox Signal 2017;27:715-726.

41 De A: Wnt/Ca ${ }^{2+}$ signaling pathway: a brief overview. Acta Biochim Biophys Sin(Shanghai) 2011;43:745-756. 